\begin{abstract}
MS31 P11
Pair distribution function analysis of zeolites Jennifer E. Readman, Paul M. Forster*, John B. Parise* and Joseph A. Hriljac, School of Chemistry, University of Birmingham, Edgbaston, Birmingham, B15 2TT, United Kingdom. * Department of Geosciences, State University of New York at Stony Brook, Stony Brook, NY 11794-2100, USA.
\end{abstract}

E-mail: j.e.readman.1@bham.ac.uk

Keywords: Zeolites, Powder X-ray diffraction, Amorphization under pressure

The structural nature of many zeolites (i.e. high symmetry framework and disorder within the pores) can often lead to complicated and unsuccessful structural analysis. Conventional powder diffraction techniques which utilise Bragg scattering often fail to resolve the disorder within the pores of zeolites and related materials. However, powder diffraction data contains information about disorder and defects in the form of diffuse scattering which is often ignored. The use of real-space pair distribution function (PDF) techniques is becoming increasingly popular in the study of disordered and nanoscale materials [1-3]. The PDF has peaks corresponding to inter atomic distances. If the PDF is compared to that of a model material then information about the local structure can be deduced. This technique has also been shown to work with poorly crystalline and amorphous materials and therefore was an ideal choice for the study of the pressure-induced amorphisation of zeolites, in particular sodium zeolite-A (Na-A). The behaviour of zeolites under pressure has been of recent interest [4-6]. In particular, it has been shown by infrared spectroscopy and synchrotron X-ray diffraction that zeolites with the LTA structure undergo a phase transition to an amorphous phase with high pressure $[7,8]$. Depending on the type of zeolite studied this phase change was found to be reversible, however, little is known about the mechanism involved in these transformations. Here we present some preliminary PDF results on series of zeoliteA samples which have been exposed to high pressure to investigate the amorphisation mechanism.

[1] Billinge, S. J. L.; Kanatzidis M. G. Chem. Commun. 2004, 749 .

[2] Proffen, Th.; Billinge, S. J. L.; Egami, T.; Louca, D. Z. Kristallogr. 2003, 218, 132.

[3] Egami, T.; Billinge, S. J. L. Underneath the Bragg peaks: structural analysis of complex materials, Pergamon Press Elsevier, Oxford England, 2003.

[4] Lee, Y.; Hriljac, J. A.; Parise. J. B.; Vogt T. Am. Mineral., 2006, 91, 247.

[5] Colligan, M.; Lee,Y.; Vogt, T.; Celestian, A. J.; Parise, J. B.; Marshall, W. G.; Hriljac, J. A. J. Phys. Chem. B, 2005 109, 1520.

[6] Lee, Y.; Hriljac, J. A.; Parise, J. B.; Vogt, T. Am. Mineral., 2005, 90, 252.

[7] Huang, Y. E.; Havenga, A. Chem. Phys. Lett., 2001, 345, 65.

[8] Rutter,M. D.; Secco, R. A.; Huang, Y.; Wang Y. J. Phys. Chem. Solid, 2001 62, 599.

\section{MS31 P12}

Relation between microstructure and synthetic procedure of $\mathrm{Ln}_{2} \mathrm{TO}_{5}(\mathrm{Ln}=\mathrm{La}$ and $\mathrm{Nd}, \mathrm{T}=\mathrm{Ge}$ and $\mathrm{Si})$ Laura León-Reina, Enrique R. Losilla, Miguel A. G. Aranda. Departamento de Química Inorgánica,
Cristalografia y Mineralogía, University of Málaga, Málaga, Spain. E-mail: 1auralr@uma.es

Keywords: $x$-ray and neutron powder diffraction, microestructure characterization

Rare earth oxy-apatites are nowadays interesting due to their high oxide ion conductivities and low activation energies, properties that are making these materials promising electrolytes for solid oxide fuel cells, SOFCs [1]. An attractive system related with the apatite-type phases is the monoclinic $\mathrm{La}_{2-\mathrm{x}} \mathrm{GeO}_{5-3 \mathrm{x} / 2}$ series, synthesized and studied by Ishihara et al [2]. The structural formula of this series is better expressed as $\mathrm{La}_{2-\mathrm{x}}\left(\mathrm{GeO}_{4}\right) \mathrm{O}_{1-3 \mathrm{x} / 2}$ to highlight its oxy-germanate character. In this system the basic conduction mechanism is likely associated with weakly bounded oxygens.

In the present communication we expand Ishihara's study to other monoclinic compounds: $\mathrm{La}_{2} \mathrm{GeO}_{5}, \mathrm{Nd}_{2} \mathrm{GeO}_{5}$, $\mathrm{La}_{2} \mathrm{SiO}_{5}$ and $\mathrm{Nd}_{2} \mathrm{SiO}_{5}$. We have used different synthetic procedures (solid state synthesis and liofilization [3]) in order to enhance the conductivity values and to reduce the sinterization temperature. We will present a discussion about the relationship between the microstructure and the synthetic procedure, using strictly monochromatic X-ray diffraction. Furthermore, the relation between oxide conductivity and crystal structure, using neutron powder diffraction, will be reported.

[1] León-Reina L., Losilla E. R., Martínez-Lara M., Bruque S., Llobet A., Sheptyakov D. V. and Aranda M. A. G., J. Mater. Chem., 2005, 15, 2489.

[2] Ishihara T., Arikawa H., Akbay T., Nishiguchi H. and Takita Y., J. Am. Chem. Soc., 2001, 123, 203.

[3] Martín-Sedeño M. C., Marrero-López D., Losilla E. R., Bruque S., Núñez P. and Aranda M. A. G., J. Solid State Chem., 2006, 179, 3445.

\section{MS31 P13}

Insulin derivative measurements on a laboratory powder diffractometer

Stjepan Prugovečki ${ }^{\mathrm{a}}$, Biserka Prugovečki ${ }^{\mathrm{b}}$, Detlef Beckers ${ }^{\mathrm{a}}{ }^{\mathrm{a}}$ Panalytical B.V., Almelo, The Netherlands.

${ }^{a}$ Department of Chemistry, University of Zagreb, Croatia.

E-mail: Stjepan.Prugovecki@panalytical.com

Keywords: insulin derivative, powder X-ray diffraction, X-ray optics

After successful structure refinement of lysozyme from laboratory powder data [1], we continued experiments with more sensitive sample. Crystals of bromo-derivative of insulin have been obtained by the batch crystallization method and loaded into the glass capillary. The capillary has been mounted onto the X'Pert PRO powder diffractometer configured in focusing transmission geometry. The data were collected in series of one hour scans in order to monitor sample decay. Several data sets from several ssmples were collected The quality of obtained data was sufficient for crystallographic investigation.

Indexing, full-profile fit and an attempt to refine crystal structure will be discussed.

[1] S. Prugovecki, D. Beckers, T. Degen, B. Prugovecki , Acta Cryst, A61, 2005, C54 